\title{
VALIDAÇÃO DO PERFIL DE VELOCIDADE DO ESCOAMENTO DE UM DUTO CIRCULAR UTILIZANDO O SOFTWARE DE SIMULAÇÃO ANSYS CFX
}

\author{
V. F. A. C. LADEIA $^{1}$, R. S. CARVALHO ${ }^{1}$, A. C. M. QUEIROZ ${ }^{1}$ e J. C. GONÇALVES ${ }^{1}$ \\ ${ }^{1}$ Instituto Federal do Norte de Minas Gerais - Campus Montes Claros \\ E-mail para contato: ladeiavictor@gmail.com
}

\begin{abstract}
RESUMO - Este trabalho compara os perfis de velocidades obtidos experimentalmente (por meio da medida das alturas manométricas por tubo de Pitot) e simulados pelo software ANSYS CFX 17.2 para o escoamento de ar em um duto de um módulo de calibração. Seis pontos do duto, variando da parede inferior ao centro, foram selecionados para aplicação dos cálculos. Os resultados numéricos e experimentais mostraram que existe uma diferença de $17 \%$ quando os pontos eram tomados próximo da parede do duto. À medida que os pontos de medição se aproximaram do centro do duto, essa diferença chegou a ser reduzida a até $1,6 \%$. A simulação se mostrou uma aliada à realização de práticas de fenômenos de transporte. Os resultados indicaram que o tubo de Pitot não estava instalado corretamente, pois quando era acusado que o ponto de medição estava localizado na parede do duto, na verdade ainda captava variações a uma distância significativa da parede, justificando o alto erro para esse ponto.
\end{abstract}

\section{INTRODUÇÃO}

Nas ciências de engenharia é de extrema importância qualificar e compreender os efeitos de determinado fluido em escoamento seja para evitar transtornos e prever seu comportamento ou até mesmo para aquisição de dados para efetuar o controle sobre determinado processo. Uma forma de estudar o comportamento do fluido é através do perfil de velocidades.

O perfil de velocidades em um duto pode ser obtido através de medidores de vazão. As velocidades podem obtidas em diferentes pontos no diâmetro do duto por meio do tubo de Pitot. Esse tipo de instrumento não percebe variações rápidas da velocidade e fornece a velocidade média para tempos de vários segundos (Bird et al., 2010). O tubo de Pitot mede variações de pressão no duto através de dois orifícios, um paralelo e outro perpendicular ao escoamento. A pressão P da equação de Bernoulli, Equação 1, é a pressão estática (Livi, 2010):

$$
\frac{\Delta \mathrm{v}^{2}}{2}+\mathrm{g} \Delta \mathrm{z}+\frac{\Delta \mathrm{P}}{\rho}=0
$$

onde $\mathrm{v}$ é a velocidade de escoamento, $\mathrm{g}$ a aceleração gravitacional, $\Delta \mathrm{z}$ a variação de altura do escoamento e $\rho$ a massa específica do fluido. A pressão dinâmica, mostrada na Equação 2, é 
diferença entre a pressão total, pressão quando a velocidade do fluido é igual a zero, e a pressão estática.

$$
\mathrm{P}_{\text {dinâmica }}=\frac{1}{2} \rho \mathrm{v}^{2}=\mathrm{P}_{\text {total }}-\mathrm{P}
$$

Isolando V, obtém-se a Equação 3, usada para calcular a velocidade de escoamento:

$$
\mathrm{v}=\sqrt{\frac{2\left(\mathrm{P}_{\text {total }} \mathrm{P}\right)}{\rho}}
$$

A diferença de pressões na Equação 3 é medida através de tomadas de pressão conectadas entre o tubo de Pitot e uma coluna manométrica. Essa diferença de pressão é empregada na Equação 4 para o cálculo da pressão dinâmica:

$$
\mathrm{P}_{\text {total }}-\mathrm{P}=\frac{1}{2} \rho v^{2}=\mathrm{g} \Delta \mathrm{h}\left(\rho_{\mathrm{m}}-\rho\right)
$$

onde $\rho_{\mathrm{m}}$ é a massa específica do fluido manométrico.

Isolando v na Equação 4, obtém-se a Equação 5, expressão que permite calcular a velocidade através da leitura da altura manométrica:

$$
\mathrm{v}=\sqrt{\frac{2 \mathrm{~g} \Delta \mathrm{h}\left(\rho_{\mathrm{m}}-\rho\right)}{\rho}}
$$

É possível descrever o perfil de velocidade de um escoamento em um duto pelo perfil da lei de potência, Equação 6:

$$
\frac{\overline{\mathrm{v}}}{\mathrm{v}_{\text {máx }}}=\left(\frac{\mathrm{r}}{\mathrm{R}}\right)^{\frac{1}{\mathrm{n}}}
$$

A velocidade média $\bar{v}$ é calculada pela lei de potência, Equação 7:

$$
\bar{v}=\frac{2 n^{2}}{(n+1)(2 n+1)} \cdot v_{\text {máx }}
$$

n é um valor tabelado que depende do número de Reynolds, Re.

Para a aplicação em sistemas mais complexos que retratem a realidade, em geral não há soluções analíticas para a equação da continuidade e para as equações de Navier-Stokes aplicadas em conjunto (FOX et al.,2014). Assim, o cálculo numérico surge para solucionar esses problemas mais complexos. ANSYS CFX é um software para dinâmica de fluidos computacional (CFD em inglês) que combina um solucionador avançado com poderosas capacidades de pré e pós-processamento (CFX User's Manual). 
Para este trabalho foi utilizada a versão Academic 17.2 oferecida pela ANSYS com o objetivo de simular o escoamento de ar em um duto, gerar perfis de velocidades e comparar os resultados computacionais com os experimentais. Com essa comparação é possível validar a simulação e tornar o software um instrumento que auxilie o desenvolvimento da aprendizagem da disciplina Laboratório de Fenômenos de Transportes do IFNMG - Campus Montes Claros.

\section{MATERIAIS E MÉTODOS}

Para a parte experimental, foi utilizado o módulo de calibração do laboratório de Fenômenos de Transportes do IFNMG - Campus Montes Claros, Figura 1:

Figura 1 - Tubo de Pitot instalado

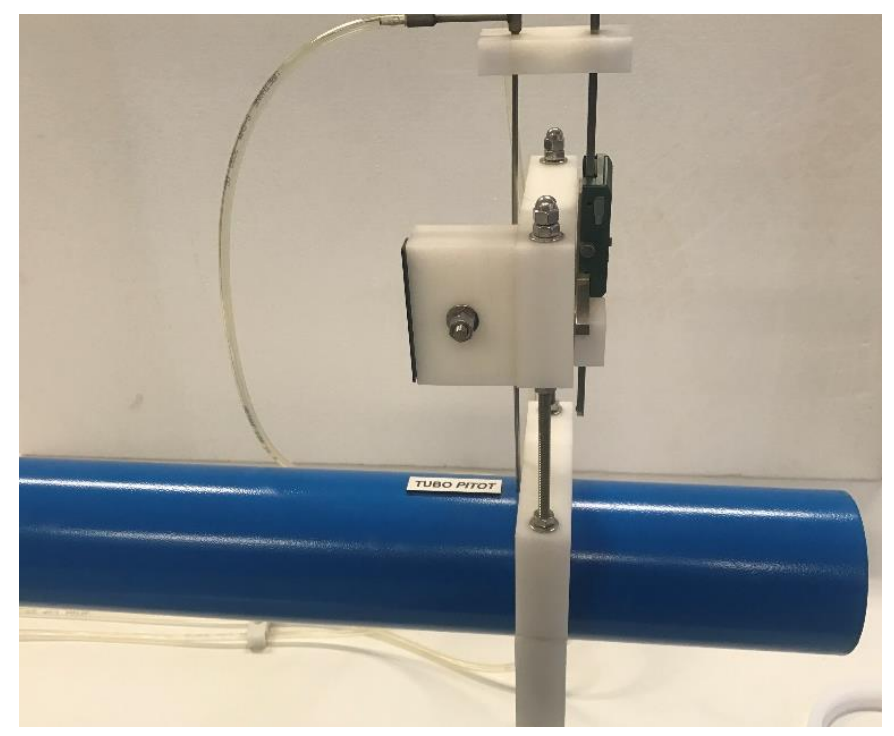

As velocidades experimentais foram obtidas com auxílio de um tubo de Pitot. A média da temperatura do fluido de escoamento, o ar, foi utilizada para determinar o valor da massa específica do ar, assim como a temperatura ambiente foi utilizada para a determinação das propriedades físicas do fluido manométrico. Os valores de massa específica e de viscosidade foram consultados em PERRY et al. (2008). Foi fixado um valor no inversor de frequência ligado ao soprador do módulo de calibração para se ter uma vazão constante. A variação da altura manométrica foi medida em triplicata para seis diferentes pontos do diâmetro do duto, variando de $0 \mathrm{a}-1$. Essa variação é referente à parte inferior do duto. Devido à geometria do tubo de Pitot, as medidas da parte superior, principalmente próximas à parede, poderiam comprometer os dados.

As velocidades experimentais foram então calculadas pela Equação 5 e a velocidade máxima foi empregada nas Equações 6 e 7.

Para a simulação, a geometria do duto foi feita no DesignModeler e a malha foi gerada no Meshing, ambos softwares da ANSYS. As mesmas dimensões do duto do módulo de calibração foram utilizadas para a construção da geometria. A malha da Figura 2 contém 21.889 nós e 108.361 elementos. A velocidade média e a temperatura média calculadas pelo experimento foram utilizadas como condições iniciais da simulação. 
Figura 2 - Malha utilizada na simulação

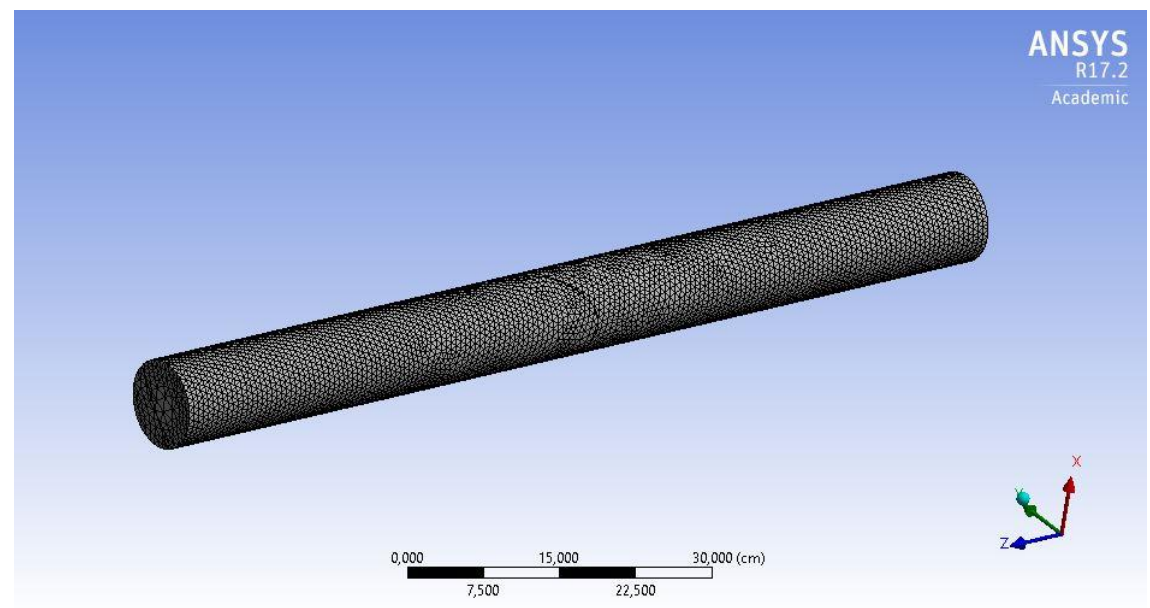

\section{RESULTADOS E DISCUSSÃO}

$\mathrm{Na}$ Tabela 1 contém os valores da variação da altura manométrica para cada ponto de medição do tubo de Pitot, além de conter as velocidades experimentais calculadas com base nessa variação e as velocidades obtidas por simulação.

Tabela 1 - Resultados experimentais e computacionais

\begin{tabular}{ccccc}
\hline Ponto & $\mathbf{r} / \mathbf{R}$ & $\begin{array}{c}\Delta \mathbf{h} \\
(\mathbf{m m})\end{array}$ & $\begin{array}{c}\text { Velocidade } \\
\text { Tubo de Pitot } \\
(\mathbf{m} / \mathbf{s})\end{array}$ & $\begin{array}{c}\text { Velocidade } \\
\text { Computacional } \\
(\mathbf{m} / \mathbf{s})\end{array}$ \\
\hline $\mathbf{1}$ & -1 & 4,25 & 15,063013 & 12,502772 \\
\hline $\mathbf{2}$ & $-0,8$ & 5,03 & 16,382401 & 15,157255 \\
\hline $\mathbf{3}$ & $-0,6$ & 5,7 & 17,43477 & 16,853611 \\
\hline $\mathbf{4}$ & $-0,4$ & 5,8 & 17,59184 & 17,309114 \\
\hline $\mathbf{5}$ & $-0,2$ & 6,67 & 18,864107 & 17,419063 \\
\hline $\mathbf{6}$ & 0 & 6,93 & 19,225368 & 17,43477 \\
\hline
\end{tabular}

A simulação foi concluída em tempo hábil, menor que um minuto. Foi gerada uma distribuição de velocidade em três dimensões. Para fim de análise, um plano foi gerado no centro do duto, facilitando a visualização da distribuição de velocidades no mesmo. Essa distribuição é ilustrada na Figura 3: 
Figura 3 - Resultado da simulação

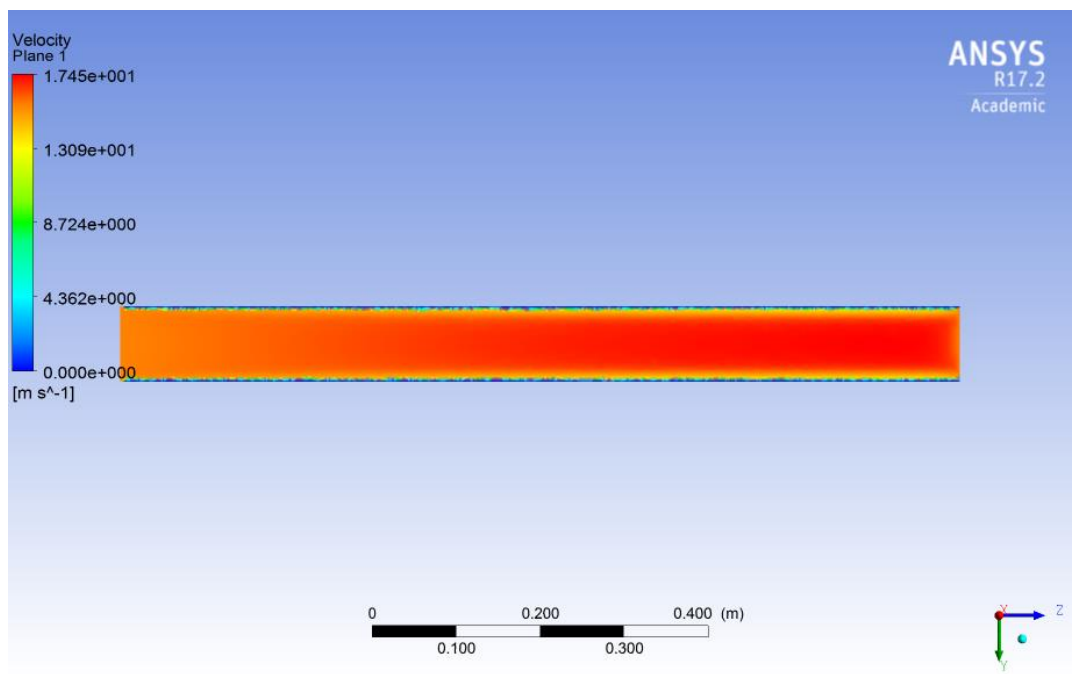

Percebe-se que somente no final, onde há cores alaranjadas mais fortes, é o local onde o escoamento se encontra em estágio completamente desenvolvido. A distribuição de cores está de acordo com o que se espera para um escoamento turbulento: pouca variação no centro do duto passando para uma variação considerável próximo às paredes do mesmo.

O valor de $\mathrm{n}$ da Equação 7 foi determinado através do número de Reynolds, encontrando o valor igual a 7. Com isso, a razão $v / \bar{v}$ foi calculada e os perfis de velocidade para os resultados experimental e computacional puderam ser construídos. A Figura 4 mostra os perfis para os dois casos:

Figura 4 - Perfis de Velocidade

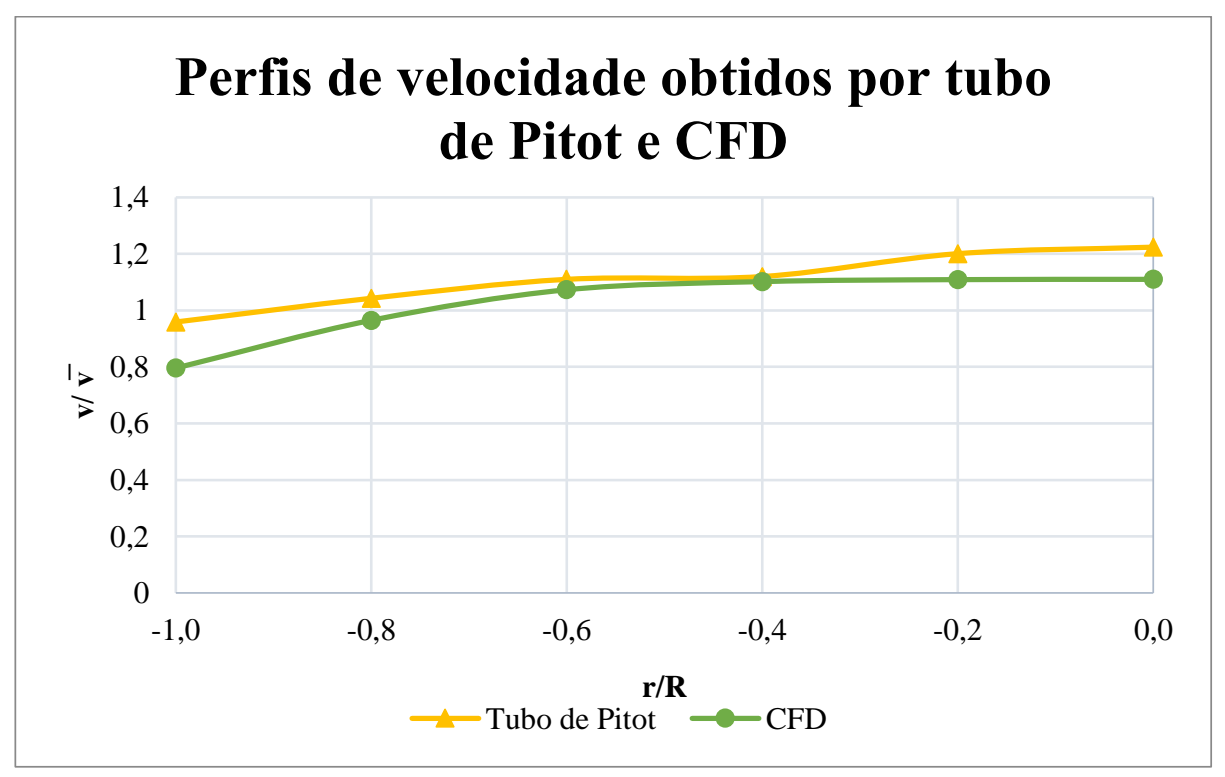

Para a razão dos raios igual a -1 , os perfis têm valores significantemente distintos. Fisicamente é impossível medir a queda de pressão em $\mathrm{r}=\mathrm{R}$. O tubo de Pitot só será capaz de 
medir as quedas de pressão satisfatoriamente, quando $r<$ R. Foi possível perceber no módulo que mesmo quando o paquímetro acoplado ao tubo de Pitot indicava que o ponto do raio era igual raio real do duto, o orifício do tubo de Pitot não se alinhava à parede do duto. Essa falta de regulagem explica a diferença de $17 \%$ em relação à velocidade computacional. À medida em que os pontos experimentais foram se aproximando do centro do duto, houve redução nessa diferença, chegando ao valor mínimo de $1,6 \%$ para $\mathrm{r} / \mathrm{R}=-0,4$.

\section{CONCLUSÃO}

A comparação de perfis de velocidades gerados experimentalmente por tubo de Pitot e computacionalmente gerados pelo software ANSYS CFX mostrou que o uso de simulações CFD são grandes aliadas aos processos experimentais. O resultado da simulação chegou a ser $17 \%$ diferente do resultado experimental. Devido a esse resultado, foi percebida a necessidade da correta instalação do tubo de Pitot no módulo de calibração usado. Conforme os pontos experimentais foram se aproximando do duto onde havia o escoamento de ar, a diferença de resultados chegou a 1,6\%, mostrando que para essa região a simulação mostrou-se uma ferramenta eficaz para a verificação da velocidade em uma tubulação.

\section{REFERÊNCIAS}

BIRD, R. B.; STEWART, W. E.; LIGHTFOOT, E. N. Fenômenos de transporte. Rio de Janeiro: LTC, 2004.

FOX, R. W.; MCDOLNALD, A. T.; PRITCHARD, P. J. Introdução à Mecânica dos Fluidos. 8 ed. Rio de Janeiro: LTC, 2014.

LIVI, C. P. Fundamentos de Fenômenos de Transportes: Um texto para Cursos Básicos. Rio de Janeiro: LTC, 2010.

PERRY, J.; PERRY, R.; GREEN, D. Perry's Chemical Engineers Handbook. 8 ed. New York: McGraw-Hill, 2008. 